\title{
Exercise induced neurally mediated syncope in an elite rower: a treatment dilemma
}

\author{
G Whyte, N Stephens, R Budgett, S Sharma, R E Shave, W J McKenna
}

Br J Sports Med 2004;38:84-85. doi: 10.1136/bjsm.2002.004507

\section{CASE REPORT}

In March 2002, a 25 year old white woman presented to the British Olympic Medical Centre after bouts of syncope and pre-syncope. The athlete was an international rower, competing in the single sculls event, and was training an average of 10 sessions/week. Training sessions included rowing and weight training, with training across the full range of intensities. The presenting symptoms included two episodes of syncope after $2000 \mathrm{~m}$ maximal tests on a rowing ergometer, together with episodes of pre-syncope on water that had not resulted in syncope. All episodes of pre-syncope/ syncope occurred immediately after exercise in a seated position. The duration of syncope was not formally documented but was described as lasting one or two minutes, with periods of malaise lasting for considerable periods (more than one hour) after syncope. There had been no previous examination of heart rate and/or blood pressure response during these episodes. Separately the patient noted a tendency for pre-syncope and syncope during medical procedures. There was no family history of heart disease including sudden "unexplained" death.

On examination, the athlete was in sinus rhythm with bradycardia (about 40 beats/min) and sinus arrhythmia. All other measures, including PR and QT interval, were within normal limits. Resting blood pressure was 110/70 mm Hg. Echocardiography showed normal intracardiac dimensions (maximum left ventricular wall thickness; $10 \mathrm{~mm}$ ) with normal systolic function of both left and right ventricles. Origins of left and right coronary arteries were normal. Mild pulmonary valve regurgitation was noted (inaudible clinically).

During integrated cardiopulmonary exercise stress testing, the athlete completed level 5 of a BRUCE protocol stopping because of maximal exercise capacity. It is recognised that the BRUCE protocol is not specific to rowing; however, it allows optimal interrogation of electrocardiogram and blood pressure during exercise. Further, the use of an ambulatory mode of exercise may better facilitate the specific symptoms reported in this case. In general, however, every effort should be made to replicate the mode of exercise associated with symptoms. The athlete achieved maximum oxygen consumption of $60.6 \mathrm{ml} / \mathrm{kg} / \mathrm{min}$ ( $185 \%$ of age and sex predicted) and a maximum heart rate of 186 beats/min. Blood pressure and heart rate response during exercise was normal. There were no inducible electrocardiogram changes or arrhythmias. On cessation of exercise, however, there was an immediate fall in heart rate ( 186 to 100 beats/min). Blood pressure measured at maximum exercise and within one minute after exercise showed a precipitate fall (200/90 to $110 / 60 \mathrm{~mm} \mathrm{Hg}$ respectively). The athlete reported symptoms of pre-syncope similar to those experienced during previous episodes. A tilt test was not used because of the non-specific nature of such a test in elite athletes.

The athlete was diagnosed with mild exercise induced vasovagal syndrome. This condition may be considered benign; however, the rower in this case study was an international calibre single scull rower, thus the potential for a syncopal episode on water and subsequent immersion and drowning left us with a treatment dilemma.

\section{DISCUSSION}

Syncope is the transient loss of consciousness and postural tone. It rarely occurs during exercise, but when it does, it is often an ominous sign of structural heart disease. ${ }^{1}$ In contrast, however, recurrent idiopathic syncope has been described after exercise in patients with normal hearts ${ }^{2}$ and has been reported to be particularly prevalent in endurance trained athletes. ${ }^{3}$

Rowing performance is associated with both aerobic and anaerobic capacity ${ }^{4}$ resulting in unique haemodynamic alterations during exercise. In addition to the aerobic contribution resulting in high cardiac outputs, the athlete works against a high resistance leading to transient increases in systolic blood pressure as high as $200 \mathrm{~mm} \mathrm{Hg}$ at the beginning of the catch phase of the stroke. ${ }^{5}$ This combination of isotonic and isometric contractions probably underpins the large cardiac dimensions observed in rowers. ${ }^{6}$

A number of recent studies have described exercise associated collapse after ultraendurance activity. ${ }^{7}$ Further, the symptoms reported may simply represent post-exercise hypotension. The episodes of syncope reported by the athlete in this case study occurred after short (less than seven minutes) high intensity exercise in a thermoneutral environment $\left(18{ }^{\circ} \mathrm{C}\right)$ and in the absence of appreciable dehydration. Our work with elite rowers over a 16 year period suggests that syncope after exercise, irrespective of intensity, is uncommon. This fact, combined with the reported pre-syncope after less exhaustive exercise and syncope during medical and dental examination, led us to believe that this was not exercise associated collapse or simple post-exercise hypotension, but exercise induced neurally mediated syncope.

The pathophysiological mechanisms of exercise induced vasodepressor syncope are incompletely understood. Early work examining baroreceptor control in athletes suggested that blood pressure control was impaired as a result of a depressed carotid baroreflex sensitivity. ${ }^{8}$ More recent work $^{3}$ has identified stroke volume as being of equal importance in the "triple product" of blood pressure control (systolic blood pressure $=$ heart rate $\times$ stroke volume $\times$ total peripheral vascular resistance). Levine $e t \mathrm{al}^{3}$ suggested that endurance athletes have more compliant, distensible ventricles associated with a chronic volume load during training, and therefore a steeper slope of the Frank-Starling curve relating left ventricular filling pressure to stroke volume than nonathletes. Thus the increase in chamber compliance and steep Frank-Starling curve, and abnormal blood pressure response, facilitating the delivery of a large volume of blood to the exercising muscle, may be beneficial to the performing athlete. It may, however, be a disadvantage during 


\section{Information box}

Syncope after exercise is more prevalent in endurance trained athletes, and, in the presence of a negative cardiovascular work up, is benign. However, if the athlete is in danger of impact injury or drowning, treatment is necessary. When pharmacotherapy is unnecessary/ restricted, continuation of exercise and lower body positive pressure manoeuvres after exercise limit/eliminate the occurrence of syncope.

orthostasis, resulting in a large decrease in stroke volume when filling pressure is reduced. ${ }^{2}$

The athlete in this case study showed normal echocardiographic findings, no inducible arrhythmia during exercise, and a normal resting electrocardiogram, suggesting the absence of a pathological origin for the reported syncopal episodes. Recent evidence ${ }^{9}$ suggests that exercise related syncope is not associated with adverse outcomes in competitive athletes with a fully negative cardiovascular work up. Therefore, in the absence of cardiac disease, the athlete should not be barred from competition. Indeed, the athlete in this case study refused to cease training and competition.

Traditional pharmacotherapy for vasovagal syndrome includes $\beta_{1}$ adrenergic blocking agents, anti-arrhythmics, and plasma volume expanders. ${ }^{2}$ The international governing body for rowing (FISA) has banned the use of plasma volume expanders, both in and out of competition, and $\beta$ blockers, in competition. In addition, care must be taken when prescribing drugs that possess negative inotropic actions. Submaximal and maximal exercise capacity is reduced in normotensive, asymptomatic people after $\beta$ blocker administration. ${ }^{10}$ Irrespective of these restrictions, however, pharmacotherapy was unnecessary in the treatment of the rower in this case study.

In the absence of traditional drug treatment, and the understandable unwillingness of the rower to abstain from training and competition, a warm down programme specifically to reduce the potential for the development of vasovagal symptoms was designed. The programme was targeted at maintaining blood pressure and venous return by continuing to exercise, rather than stopping abruptly, after exercise, particularly high intensity training and competition. Clearly, this strategy may be difficult to use after maximal/ competitive exercise because of exhaustion and technical problems associated with continuing to row on water. The absence of syncope on water in this study, however, is probably due to the necessary continuation of rowing for a single scull rower. Alternative strategies, including coughing, muscle tensing, and leg crossing, ${ }^{11}{ }^{12}$ may be effective in maintaining blood pressure immediately after exercise when continuation of exercise is compromised. Since adopting the new programme, the rower has reported only moderate symptoms of pre-syncope and no syncopal episodes after exercise, including a $2000 \mathrm{~m}$ time trial.

\section{ACKNOWLEDGEMENTS}

We thank Cardiac Risk in the Young (CRY) for their support of the Centre for Sports Cardiology at the British Olympic Medical Centre.

\section{Authors' affiliations}

G Whyte, British Olympic Medical Centre, Northwick Park Hospital, Harrow, Middlesex, UK

N Stephens, R Budgett, S Sharma, R E Shave, W J McKenna, CRY

Centre for Sports Cardiology, Harrow, UK

Correspondence to: Dr Whyte, British Olympic Medical Centre, Northwick Park Hospital, Harrow, Middlesex, UK;

greg.whyte@boa.org.uk

Accepted 16 June 2003

\section{REFERENCES}

1 Colvicchi F, Ammirati F, Biffi A, et al. Exercise-related syncope in young competitive athletes without evidence of structural heart disease: clinical presentation and long-term outcome. Eur Heart J 2002:23:1 125-30.

2 Grubb B, Temesy-Armos P, Samoil D, et al. Tilt table testing in the evaluation and management of athletes with recurrent exercise-induced syncope. Med Sci Sports Exerc 25:24-8.

3 Levine B, Lane L, Buckey J, et al. Left ventricular pressure-volume and FrankStarling relations in endurance athletes: implications for orthostatic tolerance and exercise performance. Circulation 1991;84:1016-23.

4 Ingham S, Whyte $G$, Jones $K$, et al. Determinants of rowing performance in elite rowers. Eur J Appl Physiol 88:243-6.

5 Clifford P, Hanel B, Secher N. Arterial blood pressure response to rowing Med Sci Sports Exerc 1994;26:715-19.

6 Pelliccia A, Maron BJ, Spataro A, et al. The upper limits of physiologic cardiac hypertrophy in highly trained athletes. New Engl J Med 1991;324:295-301.

7 Mark A. The Bezhold-Jarish reflex revisited: clinical implications of inhibitory reflexes originating in the heart. J Am Coll Cardiol 1983;1:90-102.

8 Sneddon J, Scalia G, Eard D, et al. Exercise induced vasodepressor syncope. Br Heart J 1994;71:554-7.

9 Holtzhausen L, Noakes T. Collapsed untrendurance athlete: proposed mechanisms and an approach to management. Clin J Sport Med 1997:7:292-301.

10 Stegmann J, Buster A, Brock D. Influences of fitness on the blood pressure control system in man. Aerospace Med 1974;45:45-8.

11 Van Baak M. $\beta$-adrenoreceptor blockade and exercise: an update. Sports Med 1988:4:209-25.

12 Krediet $\mathbf{P}$, van Dijk N, Linzer $M$, et al. Management of vasovagal syncope: controlling or aborting faints by leg crossing and muscle tensing. Circulation 2002; 106:1684-9. 